\title{
Capítulo 3 \\ Isaac Goldberg, profesional del cosmopolitismo roto
}

"We were a cosmopolitan gang. There were Italians, Scotch, Irish, Bohemians, Jews and nondescripts. Here we received our first lessons in languages" (el énfasis es mío, Goldberg 1929: 355). Ese “aquî” al que se refiere Isaac Goldberg (1887-1938) son las calles del West End de Boston, un lugar donde los niños de distintos orígenes no nacían en hospitales, a pesar de que a unas pocas cuadras se levantaba uno de los más famosos del mundo en ese entonces, el Massachusetts General. Goldberg y los otros como él, según cuenta el crítico, académico, traductor y escritor, nacían en las camas de sus madres y aprendían en las calles palabras y palabrotas en otras lenguas, del mismo modo como aprendían sobre sexo en esquinas oscuras, en casas desocupadas, en burdeles. El suyo era un conocimiento de los bordes, un cosmopolitismo de aceras. Dentro de los márgenes de la ciudad, existían otros, más íntimos, donde las lenguas también se multiplicaban, como en la misma casa de Goldberg. "Most Jewish children . . . grow up more or less language minded. They belong, are born into, an internationale of tongues" (Goldberg 1933: 271). Goldberg creció escuchando y hablando el inglés bostoniano, el hebreo de las plegarias reservado solo para hombres y el ídish común que se hablaba en la casa y que usaban las mujeres para orar. Su idea del mundo se forjaba desde esos márgenes atiborrados de gente con sus lenguas.

In those days we knew nothing of boundary lines between nation and nation; of creeds that severed mankind instead of uniting it . . When, in later years, some of us began to hear about the superiority of one tongue to another -an unscientific attitude at best, which is shared by more than one teacher of languages- and about the inferiority of one race to another-scientists are not at all agreed upon the very concept of race- some of us were saved by our childhood memories from capitulating to these facile prejudices. Most of us, I am sorry to add, were not . . . (el énfasis es mío, Goldberg 1933: 271-272).

Goldberg nació varias décadas después de que se instalara en el hemisferio la noción de una raza latina enfrentada a una raza anglosajona que pronto comenzó a pensarse superior a la primera ${ }^{1}$. Durante su niñez estas ideas ya eran

1 Según Michel Gobat, el término de raza "latina" surgió en Europa a comienzos del siglo XIX "when the rise of romantic nationalism and scientific racism led Europeans to identify their nations with races and languages" (Gobat 2013: 1348). Alrededor de la década de 1830, mientras Estados Unidos amenazaba con expandirse sobre tierra mexicana, los intelectuales franceses popularizaron el término "latinoamericanos" para nombrar a los habitantes de las antiguas colo-

Ә Open Access. (C) 2021 Soledad Marambio, published by De Gruyter. (c) BY-NC-ND This work is licensed under the Creative Commons Attribution-NonCommercial-NoDerivatives 4.0 International License. https://doi.org/10.1515/9783110749984-005 
comunes y aunque se debatían sus bases científicas, biológicas, su validez parecía bastante extendida entre los estadounidenses. Incluso años después, en 1941, Pedro Henríquez Ureña, uno de los editores de la Pan American Poetry, esa revista de tan breve existencia, volvía a Estados Unidos nombrado catedrático Norton en Harvard, haciendo hincapié en que la única manera posible de que el panamericanismo diera frutos era, primero, "modificando los paradigmas interpretativos”, como dice Fernando Degiovanni, y los modos culturales de ver a Latinoamérica como una región inferior ${ }^{2}$. Esos paradigmas interpretativos, que exploraremos un poco más adelante, eran derivados directos de aquella división de las razas. Dice Degiovanni que lo que más le preocupaba a Henríquez Ureña era que estas ideas preconcebidas no solo circularan entre la gente común sino también entre gobernantes, diplomáticos y académicos norteamericanos.

Goldberg, que venía desde los bordes de Boston y de las lenguas, se movía en mundo que avanzaba hacia la modernidad e intentaba navegarlo desde una subjetividad cosmopolita, universalizante. Esa idea suya de un mundo abierto se va a reflejar en un primer momento en su gusto y habilidad con las lenguas extranjeras, a las que consideraba su salvación: "Had I not gone on to highschool and begun the study of foreign languages, I should have remained in that outer darkness which surrounds all Americans who have never progressed beyond the elementary grades" (el énfasis es mío, Goldberg 1929: 357). El margen, esa oscuridad allá afuera de la que habla Goldberg, es el de un país cerrado en sí mismo, en su propia lengua y cultura, a pesar de sus ideas expansionistas. El cosmopolitismo de Goldberg es entonces uno que piensa el universalismo como una forma de quebrar esas formas de localismo nacionalista y de hegemonías raciales, de igual modo como lo articulan los escritores latinoamericanos que estudia Siskind y de los que hablamos en el capítulo anterior. Esos cosmopolitas de los bordes latinoamericanos desarrollan un cosmopolitismo que, en realidad, dice Siskind, es un discurso imaginario que intenta sumarlos a ese mundo moderno que miran desplegarse desde su orilla lejana. Goldberg en su etapa de traductor de obras latinoamericanas, en la que coincidirá con Alice

nias españolas del hemisferio occidental. Una década más tarde, los mismos intelectuales del centro y del sur de América adoptaron la idea y con ella el nombre de Latinoamérica, para así acercarse más a Francia y para enfrentarse también al impulso expansionista de Estados Unidos, país que además se había apropiado de los términos América y americanos, que hasta entonces también habían usado las nuevas repúblicas del sur del hemisferio. Estados Unidos, por su parte, se calzó el término de raza anglosajona para diferenciarse del resto de América a la que consideraba inferior por ser mestiza. Estados Unidos se calzaba así la marca biológica, racial, de la blancura. Para más sobre este tema ver Gobat (2013) y Horsman (1981).

2 Ver Degiovanni (2015: 145). 
Stone Blackwell como veremos pronto, usa su idea de cosmopolitismo de una manera similar: para incluir en esta idea de mundo universal a otros y también a sí mismo, judío, hijo de inmigrantes criado en barrio marginal. El problema es que en el caso de Goldberg ese discurso cosmopolita, ese deseo de un mundo abierto y conectado, se piensa desde y colisiona con el paisaje de un país en proceso de construirse sobre la idea de que no necesita de otros, de que solo requiere territorios vueltos mercados o tierra anexada y gente que le sirva y que consuma lo que el tren de su modernidad produce en el camino.

He aquí el escenario para la complejidad de Goldberg, quien después de terminar la secundaria cursó un doctorado en Harvard. Allí estudió griego, alemán, español, francés, italiano, portugués y latín, aunque luego, gracias a la guía de su mentor, el profesor Jeremiah D. M. Ford, se concentró en el español y el portugués y en las literaturas de América Latina. Es decir, Goldberg se formó como un profesional de las lenguas y de la literatura y fue desde ese lugar que se acercó a la traducción, que solo practicaría por unos años. Además, escribió ficción, teatro, crónicas, música. Junto con su formación en lenguas y literaturas, Goldberg, el de las aceras cosmopolitas, fue formado en las ideas del panamericanismo, que contaba entre sus filas a Ford y a varios de sus discípulos en el departamento de lenguas romances de Harvard. Este capítulo explora estas complejidades suyas, como se encuentran y contraponen con las de Alice Stone Blackwell, con quien va a colaborar en un par de libros, y cómo se forma profesional de las letras, traductor con fines de lucro, pero también sediento de conocimiento por una región que entiende cercana desde su cosmopolitismo "innato" y desde su idea de panamericanismo, que comparte con muchos académicos que a diferencia suya siguieron el rumbo de los latinoamericanistas profesionales y quienes encontraron en el movimiento panamericano una inspiración y confirmación de sus proyectos pedagógicos e intelectuales.

\section{Mentes panamericanas}

Jeremiah D. M. Ford (1873-1958), quien sería chair del departamento de lenguas romances de Harvard por más de tres décadas, fue el mentor de Goldberg durante sus estudios en la universidad. Además, fue quien llevó a Harvard a constituirse en uno de los centros académicos de promoción del latinoamericanismo gerencial, como explica Degiovanni. Para Ford y sus discípulos, todos seguidores del panamericanismo, el español era una lengua deseable de aprender y enseñar por ser la lengua de los negocios que vendrían con la apertura de los mercados latinoamericanos, después de la inauguración del Canal de Panamá. Es decir, como dice también Degiovanni, el español como lengua de la expansión estadounidense. 
Henríquez Ureña, dominicano, cercano a Ford aunque no directamente su estudiante, sugería en el Minessota Daily en 1917 que "(for) those (students) intended to go into business, Spanish was the language to study" (Degiovanni 2015: 141) ${ }^{3}$. La lengua hispana se volvió según este programa pedagógico un instrumento para llegar más lejos en el avance hacia los mercados latinoamericanos. Pronto, la literatura producida en la región seguiría el mismo camino hacia la instrumentalización. Ricardo Salvatore explica que los businessmen estadounidenses se dieron cuenta de que para extender sus mercados y su influencia hacia las tierras vecinas del sur necesitaban conocer más y mejor la región. Para quitarles terreno a los comerciantes europeos - porque esa era la carrera, vencer a Europa- era necesario que los diplomáticos y representantes comerciales norteamericanos aprendieran no solo las lenguas sino también las tradiciones y costumbres de Latinoamérica. Ya para los años veinte estaba claro quién debía encargarse de esta tarea de preparación: "Universities and colleges had taken on the challenge of training men in foreign trade ... but the curriculum was too practical and lacked content in the humanities. Greater training in the history and literatures of the world was required" (Salvatore 2016: 23). En consecuencia, la literatura se vuelve útil, instrumento, y es la academia la que asume la tarea de presentarla así y ponerla en uso.

En 1916, Alfred Coester, brooklyniano y discípulo de Ford escribió la primera historia de la literatura latinoamericana. Lo hizo en inglés y desde la academia estadounidense. En The Literary History of Spanish America Coester cubrió, en casi 500 páginas, desde la colonia hasta el movimiento modernista y trató de recorrer la mayor cantidad de países y de escritores y estilos posibles ${ }^{4}$. Su idea, allí, es leer la literatura como hechos, facts, dice cuando cita y a la vez traduce al ex presidente argentino Bartolomé Mitre quien había advertido que la Latinoamérica de comienzos del siglo XX aún no tenía una literatura, sino un conjunto de textos que podían leerse como hechos, o información. Para Coester, como para Ford y el resto de los estudiantes formados en el latinoamericanismo gerencialista, lo importante de la literatura era que permitiera aprender, entender. Por eso, dice que leyendo su libro "[an English-speaking American] will better comprehend the difference between the sober energetic Chilean and the fun-loving Peruvian, or the

3 Ford recomendó a Henríquez Ureña como lector y estudiante de posgrado en la Universidad de Minnesota en 1916. Luego, en 1940, lo invita a Harvard como catedrático Norton, en un nombramiento inusual para una cátedra que solía tener figuras de la talla de T. S. Eliot e Igor Stravinski. El nombramiento de Henríquez Ureña tuvo que ver con la Política del Buen Vecino, que intentaba "sumar aliados en el campo intelectual para impulsar la expansión económica hemisférica” (Degiovanni 2015: 136).

4 Más tarde, Henríquez Ureña le criticará no haber hecho distinciones entre escritores menores y mayores. 
passionate Venezuelan. He will understand why there have been so many revolutions in Mexico. . ." (el énfasis es mío, Coester 1916: x). Comprender, aprender sobre esos vecinos del sur y sus diferentes caracteres, esa es la propuesta. "The ultimate result in improving relations between the Americas by dispelling ignorance is incalculable”, diría Coester unos años más tarde ${ }^{5}$.

Esta idea de la literatura - de cierta literatura, la elegida por esas mentes lectoras y educadoras que la importará y explicará en Estados Unidos- como vehículo de la comprensión extiende su influencia hacia los márgenes del campo ocupados por la traducción, que también se vuelve herramienta en manos de estas mentes del movimiento panamericano. Esta tendencia se iría asentando en el tiempo, impulsada luego por la Política del Buen Vecino y su propuesta de intercambio cultural como modo de ampliar la zona de influencia del panamericanismo ${ }^{6}$. Durante los años de la Segunda Guerra Mundial, cuando de nuevo una Europa en crisis se ausenta de los mercados latinoamericanos, esta percepción instrumentalista de la literatura latinoamericana y sus traducciones recobrará fuerza. En 1942, Sumner Welles, subsecretario del presidente Roosevelt y uno de sus consejeros en política exterior, escribió lo siguiente en un folleto de promoción de las traducciones de textos latinoamericanos de la casa Knopf:

The works from the Latin American republics which will have the widest appeal in this country are recent volumes on inter-American or international affairs and novels. And it is perhaps in the field of novels that the greatest benefit will result from the standpoint of inter-American relations for the novel which deals with the character and the individual manner of being of each American people necessarily affords to its readers the easiest and, in many ways, the most effective method of getting the "feel," and understanding the life, the national customs, and the problems of Central and South America (el énfasis es mío, Livingstone 2015: 12) ${ }^{7}$.

Como se ve, las ideas que comienzan a forjarse en torno a Ford y los suyos conservan su aliento a lo largo de la era del panamericanismo. La novela se ha convertido en el género de recolección de información por excelencia y también en

5 Parte del artículo "Practical Pan Americanism”, que Coester preparó para el Congreso Panamericano de Panamá, de 1926 (conmemorativo del congreso convocado por Bolívar en 1826). Ver Coester (1927).

6 Ver Fox (2013).

7 Welles escribe esa presentación en el folleto a pedido de Blanche Knopf quien viajó a Latinoamérica a buscar obras que traducir gracias a la ayuda de Welles y del gobierno estadounidense que veía con buenos ojos la cooperación intelectual inter-americana. Knopf está entre las primeras, pero no es la única editorial que vuelca su interés en Latinoamérica cuando la Segunda Guerra Mundial complica los viajes y el intercambio con Europa. Para más sobre esto ver Livingstone (2015). 
el lugar donde abundar en las ideas sobre los caracteres nacionales y las diferencias raciales que estaban en boga ya para los tiempos del nacimiento de Goldberg. Es la academia estadounidense que se especializa en Latinoamérica la que sirve para amplificar y extender estas ideas sobre la literatura y sobre lo que trae sobre la gente de la región. En otros frentes se hacía lo propio:

Whether they were staunch supporters of Pan Americanism or not, scholars tended to envision the nature of the U.S. hegemony in South America as something to be wrought in the terrain of culture. In this regard, their views sustained and accompanied the transition from Big Stick diplomacy to the Good Neighbor Policy (Salvatore 2016: 6).

Los años de Ford, Coester y Goldberg, entre otros, fueron los años formativos. El tiempo en que estas ideas se forjaron eran aún los de la 'Big Stick diplomacy', cuando Theodore Roosevelt promovía su famoso "speak softly, and carry a big stick", que lo llevaría entre otras cosas a quedarse con el proyecto del Canal de Panamá y a expandir su dominio sobre $\mathrm{Cuba}^{8}$. Fue entonces que en Estados Unidos comenzaron a explorar formas de relacionarse con las elites intelectuales latinoamericanas. La academia y sus investigaciones avanzaban junto con las políticas exteriores del país y, como dijimos en el "Interregno" anterior, muchos de los académicos involucrados estaban orgullosos de que sus conocimientos, su trabajo sobre América Latina, sirviera para conformar las políticas para con la región. Incluso, algunos académicos como Coester trabajaron directamente para el gobierno de Estados Unidos. En 1942, John T. Reid contó en un artículo que homenajeaba en vida a Coester, que en 1919 "(Coester's) special linguistic talents were called into service by the United States Department of State" (Reid 1942: 264). El discípulo de Ford fue enviado a Chile, Argentina y Brasil como investigador de la American Geographic Society, cargo gracias al cual pudo recopilar "valuable information" acerca de la colonización alemana en los llamados "ABC countries" e investigar la relación de estos colonos con la guerra. Siguiendo el trabajo de Helen Delpar me gustaría enfatizar el hecho de que fue solo un puñado de académicos relacionados con el estudio de Latinoamérica quienes trabajaron directamente para el gobierno en la época que tratamos. Otros muchos, dice Delpar "had the opportunity to express their views regarding contemporary U.S.-Latin American relations in public lectures and in books and articles addressed to general readers as well as specialists” (Delpar 2008: 91).

8 Concretamente, Estados Unidos anexa parte del territorio, Guantánamo, y fuerza una serie de acuerdos comerciales que no benefician a la isla. 


\section{El intento de profesionalizarse: ¿traductor o traditore?}

"It became evident, when at last I left Harvard as a Ph.D. in Romance Languages, that I was not cut out to be a teacher” (Goldberg 1933: 272), recordaría Goldberg poco más de dos décadas después de terminar sus estudios en Harvard ${ }^{9}$. Aunque Goldberg no haya querido seguir la vida de la academia, no es claro que le hubiera sido fácil obtener y mantener un puesto en ella en los años previos a la Primera Guerra Mundial. A fines de la primera década del siglo XX mientras universidades como Columbia y la New York University mostraban incomodidad con la gran presencia de estudiantes judíos, Harvard era reconocida por ser amable y hospitalaria con ellos. Aunque esa tendencia oscilaría con el tiempo -Sussane Klingenstein cuenta que en 1922 el entonces presidente de la universidad intentó establecer una cuota de estudiantes judíos- Goldberg siempre gozó del favor de su mentor ${ }^{10}$. Goldberg había sido uno de los mejores estudiantes de Ford y su influencia se dejó sentir aun en el camino no estrictamente académico que el antiguo discípulo decidió tomar ${ }^{11}$. Para comenzar, fue Ford quien llevó a Goldberg a especializarse en el estudio y la divulgación de la literatura latinoamericana. En 1913, después de un viaje que emprendió a Sudamérica como representante de las universidades estadounidenses y que lo llevó a conocer las principales instituciones académicas de la región, Ford le ofreció a Goldberg que escribiera una serie de artículos sobre Latinoamérica que le habían encargado a él para The Bookman ${ }^{12}$. Alegó estar muy cansado para ocuparse de ellos y que Goldberg era la persona perfecta para encargarse de la tarea. Después

9 Goldberg termina su doctorado en 1912, el mismo año en que Ford asume como chair del Departamento de Lenguas Romances.

10 Klingenstein cuenta que después de 1880 el número de estudiantes judíos matriculados en las universidades (colleges) de Estados Unidos tuvo un aumento explosivo. Dice: "A survey of 77 institutions carried out by the Immigration Commission in 1908 found that 8.5 percent of the male student body was composed of first- and second-generation Jews at a time when Jews made up only 2 percent of the American Population” (Klingenstein 1998: 6). Poco más de una década más tarde comenzarían los intentos por establecer cuotas, veladas o no, para la matrícula de estudiantes judíos. En cuanto a los profesores judíos en la academia estadounidense, como muestra Klingenstein, eran pocos antes de la Segunda Guerra Mundial y los que llegaban a ella tenían que romper una serie de barreras impuestas para evitar su presencia en la mayoría de las universidades.

11 Frederick C. H. García asegura que Goldberg se destacó por ser uno de los mejores estudiantes que tuvo J. D. M. Ford. Ver García (1972).

12 The Bookman fue una revista literaria publicada en Nueva York que circuló entre $1895 \mathrm{y}$ 1933. Allí, por primera vez, se publicaron las listas de best sellers de Estados Unidos. Goldberg fue un colaborador frecuente. 
de eso, The Boston Evening Transcript comenzó a pedirle a Goldberg artículos sobre literatura, pero también sobre música y teatro. "I didn’t know it, but I was launched”, diría después Goldberg (Goldberg 1933: 272). Sin darse cuenta, había comenzado su especialización en la literatura latinoamericana ${ }^{13}$. La cantidad de artículos que le pedían sobre las letras de la región aumentó con la apertura del Canal de Panamá y con el estallido de la Primera Guerra: “The national interest at the time, was great" (Goldberg 1933: 272). El orden geopolítico estaba cambiando, Europa se volvía sobre sí misma para revisar sus heridas y seguir un conflicto al que aún le faltaban años y millones de muertos. Latinoamérica era vista como una tierra fuera de la guerra y, sobre todo, un lugar de mercados por explorar ahora que las potencias europeas no podían ocuparse de comerciar con la región. Estados Unidos sentía la oportunidad que se le presentaba. El panamericanismo se preparaba para su avance. Así, Goldberg se encontró de pronto escribiendo sobre la región y traduciendo sus letras -y también del ídish y del italiano, aunque en menor medida-, a un ritmo desenfrenado. A los periódicos ya nombrados se sumaron otros, incluyendo The Stratford Journal, que editaba el mismo Goldberg con uno de sus amigos de la infancia, H. T. Schnittkind, otro niño de los bordes que había llegado hasta Harvard. Dice Goldberg sobre esos años de trabajo frenético:

Soon I was doing numerous translations from many tongues. The list would be too long for this brief reminiscence. Under the circumstances - hurry, poor pay, uncertainty, crowded hours- much of this activity was the toil of the hack . . . Some of the material deserved no better treatment than it received. It is disgusting to repent . . . of work done in haste, - to see mistranslation, or careless translation, when one knows that one is much superior to this ineradicable cold print- . . . (el énfasis es mío, Goldberg 1933: 273)

Una traducción tras otra. Por dinero, por poco dinero, pero de todas maneras por un pago y con tiempos de entrega y textos que caían uno sobre otro. Goldberg se hizo traductor como respuesta a esa necesidad de ocupar la literatura como herramienta de conocimiento en el gran plan de la expansión norteamericana. Como él mismo escribe, mucho del material que tradujo no le interesaba o le parecía de mala calidad, pero de todas maneras aceptaba traducirlo. Era su trabajo, su momentánea profesión, y no una cualquiera sino una que tenía que ver con uno de sus placeres, la literatura, pero que debido a las circunstancias unas que traen a la mente las condiciones actuales de muchos traductores con-

13 Entre su producción más destacada de esta época se cuentan sus Studies in Spanish American Literature (1920), Brazilian Tales (1921) y Brazilian Literature (1921). 
temporáneos ${ }^{14}$ se transformaba en "the toil of the hack": el trabajo duro de una persona "who hires himself or herself out to do any kind of literary work; (hence) a writer producing dull, unoriginal work", según una de las acepciones de "hack" del Oxford English Dictionary. Goldberg traducía lo más rápido, lo más transparentemente posible (como veremos con profundidad en el capítulo siguiente), como si no dejara, la mayoría de las veces, su huella en su escritura. Así, ¿podemos pensarlo como un traductor literario? Para explorar la respuesta a esta pregunta volvamos a la idea del amateur, del que ama, y detengámonos en la definición que hace Esther Allen de la traducción literaria:

Literary translation is the re-embodiment of a text within the lived experience and erudition of a translator, using rational thought, sense memory, nostalgia, yearning and a host of other conscious and unconscious factors to negotiate among shifting resonances of meaning that echo against and through a given culture at a given moment. (el énfasis es mío, Allen 2013: 214)

Con esto volvemos a la idea que ya habíamos discutido antes, la traducción como interpretación y, sobre todo, como expresión de deseo. ¿Cómo se le asigna un valor a ese trabajo? ¿Cómo se retribuye materialmente esa preparación intangible, sutil, que sin duda forma la experiencia de un traductor y la forma en que esta o este se acerca a un texto? Pareciera más fácil pagar el trabajo mecánico, pretender que la traducción no es interpretación sino el intento de trasvasijar un texto de una lengua a otra, palabra por palabra, equivalencia semántica que dejaría afuera tanto. Sin embargo, esa traducción transparente es la única imposible, por lo menos cuando se piensa en literatura. Goldberg recibe algunos textos como si estos estuvieran muertos y, en esos casos, traduce como si desapareciera en ese trabajo forzado que le sirve para pagar la renta y sostener la familia. No quiere filiación con esas traducciones que no nacen del placer ni de la libertad de la selección. Aunque debe haber habido uno que otro encuentro fortuito con algún texto desconocido de antemano que lo haya conquistado después de una lectura. Además, sin duda hubo otros que buscó por gusto personal e impulsado por esa idea cosmopolita que lo animaba desde niño porque, como él mismo dijo, "On the other hand, many a page that I did in these years still gives me pleasure: the feeling that one was part of a movement that opened windows

14 Dice Venuti que la mayoría de los traductores independientes debieron enfrentar durante el siglo XX -y tranquilamente agreguemos el XXI- una situación marcada por “. . . belowsubsistence fees (that) force them either to translate sporadically, while working at other jobs (typically editing, writing, teaching), or to undertake multiple translation projects simultaneously, the number of which is determined by the book market and sheer physical limitations" (Venuti 2008: 10). 
upon the far-flung corners of the world" (el énfasis es mío, Goldberg 1933: 272). El placer rondaba su práctica de traducción, ciertos textos que lo hacían sentirse conectado a un mundo más grande, imposible de imaginar sin la intervención de traductores, de intérpretes de textos de otras culturas, de otras lenguas. ¿Qué pesaba más?, ¿ese placer ocasional o esos textos casi despreciados que traducía con prisa y sin prestar mucha atención?, ¿cómo afectaba eso su práctica de traducción, profesionalizada, puesta a correr dentro de los límites del sistema de producción capitalista, lejos de la idea del amateur, de Stone Blackwell, quien será estrecha colaboradora de Goldberg?

\section{Traductor a trato}

Querido colega:

Me ha sorprendido muy gratamente su carta . . . en la cual usted me hace el honor de proponerme traducir al inglés alguna de mis obras . . . Porque su carta me revela que no es usted un traductor de tres al cuarto sino un escritor de pluma experta y brillante que no quiere traducir al primer autor que le cae en las manos sino a los escritores con cuyo espíritu siente afinidad y a quienes ya ha juzgado como crítico (Isaac Goldberg Papers $)^{15}$.

Esta carta que en 1919 Rufino Blanco Fombona, escritor y político venezolano, le escribe a Goldberg desde su exilio en Madrid es parte de un intento enorme que el traductor bostoniano hizo por asegurarse un buen número de obras para traducir y comercializar. Blanco Fombona tenía algo de razón, Goldberg quería traducir a ciertos autores por los que sentía afinidad o admiración -por ejemplo, el mismo venezolano, quien será uno de los pocos autores estudiados en el libro sobre los modernistas que Goldberg prepara durante el fin de la Primera Guerra Mundial y que tendrá las traducciones de Alice Stone Blackwell-, pero además buscaba cantidad. "The toil of the hack", como dijimos. Goldberg quería traducir y ganar dinero, transformar la práctica en profesión y no solo en un oficio amateur. Se había formado en la escuela y en la universidad en lenguas y literatura y quería capitalizar eso -su formación de profesional, realizada en las instituciones pertinentes- por fuera de la academia. Por eso también en 1919 le escribió a John Garret Dunhill, el representante en Estados Unidos de la sociedad de autores españoles, para asegurar los derechos de traducción de los trabajos de “todos los artistas destacados de España” (Isaac Goldberg Papers) ${ }^{16} \mathrm{y}$

15 Rufino Blanco Fombona a Isaac Goldberg, 26 de junio de 1919, TS. Isaac Goldberg Papers. 16 El énfasis es mío. John Garret Dunhill a Isaac Goldberg, 10 de marzo de 1919, TS. Isaac Goldberg Papers. 
mientras pensaba cómo conseguirlos le volvió a escribir a Blanco Fombona para pedirle la exclusividad de todas sus obras y también las de la editorial América que este dirigía en Madrid. El venezolano le contestó que le entregaría el derecho a traducir la totalidad de sus obras, pero que prefería tener más cuidado con los textos que publicaba su editorial, la que entre 1915 y 1933 puso en circulación más de 400 obras de escritores latinoamericanos. Blanco Fombona le ofreció a Goldberg prioridad con los títulos publicados por América, pero le reservó solo doce al año diciendo que no quería comprometer una cantidad mayor porque temía que de otra manera las traducciones serían hechas de manera descuidada o por terceros no tan diestros como Goldberg, quien para Blanco Fombona era el mejor traductor al inglés que había conocido. Esto, porque Goldberg además de tener formación en las lenguas, tenía pluma. Su escritura es ágil, cuidada, honda, y así lo había notado el escritor venezolano. En eso, lo sentía cerca. Además, los dos coincidian en la necesidad de que su producción literaria fuera bien remunerada, como le dice Blanco Fombona al traductor en una carta de 1919: "Tanto lo que usted como yo debemos procurar es que los beneficios económicos para ambos, lo mismo que la seguridad de cobranza respecto a los beneficios, no sean una quimera. . .” (Isaac Goldberg Papers) ${ }^{17}$. Para Blanco Fombona y para Goldberg, por lo menos en el momento de este intercambio, la escritura y la traducción son profesiones, actividades a las que se les dedica tiempo y recursos y por las que se espera recibir retribución monetaria. Esto es, la escritura, la del centro y la del borde, como mercancía. 0 , por lo menos, ese era el intento.

Como dijimos hace un momento, Ford también se hizo presente en esta etapa de la sinuosa carrera de su pupilo. En 1918, le escribe a Goldberg para agradecerle por la noticia de que sus cuentos brasileños en traducción -Brazilian Tales, publicados finalmente en 1921- van a estar dedicados a él y de paso lo felicita por la labor de educación que está emprendiendo con la preparación de su Studies in Spanish-American Literature (1920), un libro dedicado al estudio de los modernistas latinoamericanos, dentro de quienes Goldberg destacará a Darío, Blanco Fombona, Santos Chocano, Rodó y Eguren, además de otros a quienes presenta como precursores como Martí, Gutiérrez Nájera, Julián del Casal y José Asunción Silva. Además, Ford le recomienda a Goldberg una serie de textos literarios para traducir, entre ellos las Tradiciones peruanas de Ricardo Palma, Amalia de José Mármol y alguna novela de Alberto Blest Gana. Un año después el antiguo mentor vuelve a escribir, esta vez para calmar la ansiedad que parece tener Goldberg sobre las posibles ventas del libro que ya se apresta a terminar. Ford le dice:

17 Blanco Fombona a Isaac Goldberg, agosto de 1919, TS. Isaac Goldberg Papers. 
One would expect a reasonably good sale for works dealing with Spanish-American literature and I hope that you will issue the book that you contemplate. You may be sure that I shall find a place for it in the curriculum here. It is a disconcerting thing that the sale for Dr. Coester's book remains relatively slow, but its size and price must have something to do with that. In all probability a less expensive book dealing with a limited number of leading personalities will attract greater attention. . . ${ }^{18}$ (Isaac Goldberg Papers).

A fines de la Primera Guerra Mundial los temas latinoamericanos aún gozaban de una gran popularidad en los Estados Unidos. Goldberg se aprestaba a publicar su libro en un buen momento y había pensado muy bien el tipo de volumen que quería: una mezcla de la presentación de un movimiento que mostraba transversal a gran parte de Latinoamérica, en el que se referiría a pocos autores y en el que se entregaban fragmentos de obras, algo de crítica y de historia literaria. Un texto ágil y fácil de navegar, para uso de los amateurs de las letras y de quienes se formaban como profesionales de ellas. Un libro vendible, no como el de Coester. Y Ford ya le había prometido hacerle un espacio en el currículum en su departamento en Harvard. Goldberg pensaba su emprendimiento de la presentación de la literatura latinoamericana no solo como una forma de expandir el conocimiento de los Estados Unidos sobre América Latina, sino también como una forma de ganarse la vida. Tal vez fue su idea de vender bien el libro lo que lo llevó a buscar como colaboradora, específicamente como traductora de la mayor parte de los textos extractados, a Alice Stone Blackwell. En los años que rodean al fin de la Primera Guerra, Blackwell ya contaba con un enorme capital simbólico, era conocida y respetada por parte de la población por su trabajo como editora y sufragista, y había tenido un éxito inesperado con su antología de poemas armenios en traducción ${ }^{19}$. En ciertos círculos cultivados, su nombre ya era asociado a la traducción de poesía extranjera, un hecho no muy común para esos años, menos tratándose de una mujer. Para cuando Goldberg trabaja en su libro, Blackwell ya había aprendido español, es decir, ya se había enseñado la lengua y había publicado sus traducciones de poesía latinoamericana en diversos medios de prensa estadounidense. Además, ya era conocida entre los intelectuales de Harvard que estudiaban literatura latinoamericana. De hecho, en 1919 algunas de sus traducciones de textos de José Santos Chocano habían formado parte del capítulo dedicado a Hispanoamérica de Main Currents of Spanish Literature, de Ford. Como sea que se haya forjado la colaboración entre ella y Goldberg -no hay material de archivo que lo registre- Goldberg la invita a colaborar con él y usa sus traducciones a lo largo de casi todo su libro, excepto por

18 Jeremiah D. M. Ford a Isaac Goldberg, 24 de mayo de 1919, TS. Isaac Goldberg Papers. 19 "The well known editor and suffragist", así presenta Goldberg a Blackwell en el prólogo de Studies in Spanish American Literature. 
algunos fragmentos de traducciones hechas por él de textos citados en español en sus ensayos, pero no trabajados de antemano por Blackwell. Son estos pequeños textos, los que Goldberg dice trabajar "muy literalmente”, los que demostrarán la gran diferencia entre los dos traductores que volverán a colaborar en el libro de Blackwell Some Spanish-American Poets y en una antología de poesía mexicana en traducción editada por Goldberg. Ella tiene una escritura visible, una forma de traducir que la pone en la página mientras que en Goldberg se hace presente el acto de desaparición, la invisibilidad del traductor lograda a través de la supuesta fluidez del texto traducido. De todo esto hablaremos en profundidad en el capítulo que sigue, pero lo que quiero destacar acá es que ese intento de Goldberg de ser transparente coincide con la carga enorme de traducciones que tiene a su haber y con la idea de hacer de la práctica una profesión, con horas, plazos, pago. Le dice Blanco Fombona en una carta de 1921: "Por aquí he conocido a una persona que me ha hablado de usted. Me lo pinta al frente de taquígrafos, mecanógrafos, etc. a quienes usted dicta o hace copiar y que tiene en movimiento como un general a sus tropas" (Isaac Goldberg Papers) ${ }^{20}$. Goldberg se dividía, organizaba su ejercicio de traducción y el de otros, tratando con todas sus fuerzas de sacar a la práctica de la traducción de los márgenes del mercado para poder vivir de ella. Tale vez sobra decir que no tuvo mucho éxito.

\section{Goldberg, el escritor}

Cuando Goldberg aún era un niño un diario de Boston publicó un cuento suyo. Al poco tiempo, el mismo periódico publicó un himno también compuesto por él, una canción en cuatro partes, inspirada en la música de las orquestas de las casas de burlesque. Solo, con los materiales que le proporcionaba su entorno, se construyó como escritor y compositor. Su educación literaria, dice, comenzó con las novelas de diez centavos, de las que devoraba bibliotecas enteras, y siguió con sótanos encerrados y húmedos donde se montaban espectáculos de marionetas italianas, casas de burlesque y los teatros pobres del barrio que más tarde serían reemplazados por otros menos precarios. La música la aprendió escuchando, anotando, y luego practicando con un violín viejo encontrado en el fondo de un ropero de su casa. Goldberg se enseñó a escribir música y cuentos, se formó, en primera instancia, como autodidacta, como sujeto maravillado ante el encuentro con ciertos textos, ciertas melodías que lo llevaron a aprender y practicar, lejos de cualquier institución, cerca de la calle y de lo que su intui-

20 Blanco Fombona a Isaac Goldberg, enero de 1921, TS. Isaac Goldberg Papers. 
ción le iba dictando. En este escenario, las letras siempre tuvieron un lugar más importante tanto en el uso de su tiempo como en el despliegue de sus recursos. Esto porque él mismo se declaró siempre fascinado por la complejidad de la palabra más que por cualquier otra cosa: "Words, to me -words and structure of words- are, and always have been, among the miracles of the universe" (Goldberg 1933: 272). Goldberg ponía a las palabras en uso siempre que podía. Escribía y escribía, sobre muchos temas, porque le gustaba explorar y buscar a través de la escritura "new territory . . . material that is not submerged beneath a heap of anterior opinion” (Goldberg 1933: 273). Quería originalidad, a tal punto que produjo una colección de textos diversos y abundantes en tantas áreas que es difícil hablar de "una” carrera a su haber. La traducción sería una de ellas, como se desprende de este texto de Frederick C. H. García, quien trata de explicar la razón por la cual Goldberg dejó, de pronto, antes de la llegada de la década del 30, de traducir y de escribir sobre América Latina y sus letras:

Goldberg's numerous activities have been mentioned, and in the light of them we may understand why he did not stay with Portuguese and Brazilian books, why he stopped writing about Spanish letters. Go to a library; find Goldberg's drawer; count the cards that have his name -well over a hundred of them. Translations, literary criticism, music appreciation, biographies, anthologies, editions of plays and several foreign-language anthologies, linguistics, Jewish affairs, interpretation of philosophical writing, original fiction. . . and the cards cover only the books; a lot is lost or forgotten in all kinds of periodicals. . . each subject would go through a cycle, with a moment of high interest and, without much of a transition, be all but forgotten (el énfasis es mío, García 1972: 22-23).

Goldberg tiene pasiones fugaces. Con ciertos textos, géneros, temas, voces. Escribe y escribe como si buscara algo desesperadamente. Lo que no le sirve para encontrar eso que parece estar buscando, va quedando atrás, y es bastante lo que deja atrás en los cortos 50 años que alcanza a vivir. Sus aventuras escriturales parecen una versión en fast-forward de la actitud del crítico definido por Saikat Majumdar:

The scholar is defined by his commitment to his archive of study . . . The critic on the other hand, celebrates and foregrounds his subjectivity; the archive, in his case, is subordinated to the self, through which is processed and presented, the very personal color of that refraction remaining the most cherished element of the process. In this, the critic is more closely allied to the poet or the fiction writer than to the scholar. . . (el énfasis es mío, Majumdar 2017: 7).

Goldberg pareciera acercarse más al poeta y al escritor de ficción que a sus compañeros de la academia. Escribe ficción y obras de teatro, además de crítica, ensayos, columnas sobre teatro, jazz, literatura. Pero suspende el impulso amateur al formalizar sus conocimientos y adquirir otros bajo la estructura de la universi- 
dad. Sobre todo, profesionaliza su relación con las letras -aunque no sigue el camino de la academia y continúa con su impulso de escritura prolífica, diversa-, al llevar el impulso profesionalizante a su forma de pensar y relacionarse con las traducciones de literatura latinoamericana que emprende. Al intentar volver el oficio en empresa, en productos rápidos dirigidos, sobre todo, al consumo, amparado en la idea de que podía encontrar ahí, sobre cualquier otra cosa, ganancia.

\section{Traductor panamericano, cosmopolita estadounidense}

La breve etapa de Goldberg como traductor coincidió con años clave de la formación del panamericanismo. Como ya dijimos antes, Ford, su mentor en Harvard, fue uno de los académicos asociados a este movimiento que proponía una alianza regional basada en la expansión de las relaciones comerciales. Para los académicos vinculados con el panamericanismo tanto la literatura y las lenguas latinoamericanas como el estudio de estas mismas eran herramientas para avanzar en el proyecto de unificar el continente bajo el liderazgo estadounidense, como queda claro en la breve introducción que Ford escribe para el libro de Goldberg Studies in Spanish-American Literature. Allí se lee: "What the Modernist Movement means ... Dr. Goldberg aims to make clear in the pages of this book, and, doing this, he is also playing his useful part in the spreading of the evangel of intellectual Pan-Americanism” (el énfasis es mío, Goldberg 1920: viii). Goldberg es, según la visión de Ford, uno de los apóstoles del movimiento. O tal vez deberíamos decir "misión" y pensar en la misión totalmente de otro signo que la Mistral veía en el trabajo de traductora de Blackwell (ver capítulo 2). Sin embargo, Goldberg desconfía de cualquier formación religiosa. Lo que marca su misión es de otro orden. A través de su trabajo académico, al entregar una mirada a las mentes vecinas, vuelve útil el estudio de las letras, las instrumentaliza y las presenta de modo que puedan congregar gente, seguidores, adeptos al panamericanismo. Y no cualquier adepto, sino uno que se pliegue a esa idea de que son las mentes de la región las que están en proceso de acercamiento o en peligro de distanciarse, malentenderse y malograr la unidad panamericana. La palabra es poderosa, dicen Ford y los suyos, la literatura puede acercar pueblos a través del conocimiento que entrega o puede enemistarlos. Por esto, aquellos que usan la palabra o, peor aún, la literatura, para oponerse al panamericanismo son señalados y mirados con recelo, como Darío, Rodó, y tantos otros ${ }^{21}$. A comienzos de los 20, J. Fred Rippy “encargado” del área de historia latinoamericana en la Universi-

21 Para más sobre esto ver Rippy (1922) y Pita González y Grillo (2012). 
dad de Chicago escribe un artículo titulado "Literary Yankeephobia in Hispanic America”, donde dice que:

North American students of the modern literature of Hispanic America have noted a decided element of opposition to the United States. This they have attributed to the fact that the political aspirations of the literary men south of the Rio Grande are pretty closely bound up with the fear of the United States as a possible aggressor (Rippy 1922: 350).

Los estudiantes norteamericanos que se asoman a estos textos que deberían ponerlos en contacto con el pensamiento de las elites latinoamericanas descubren en ellos el recelo, y muchas veces el desprecio, contra Estados Unidos. Los profesores de esos estudiantes también acceden a esos textos y, en ocasiones, ayudan a llevarlos hacia el público estadounidense. En 1919, mientras Goldberg alistaba su libro para imprenta, Ford le escribe una carta donde le advierte sobre los malos pasos de uno de los escritores que Goldberg ha decidido antologar: "Be careful in your dealings with Blanco Fombona. He seems to be showing a change from his attitude of hatred for all men and things of this country; but I do not trust him. . ." (Isaac Goldberg Papers) ${ }^{22}$. El escritor venezolano desde sus comienzos había dejado en claro su oposición a los avances imperialistas de Estados Unidos y su decidida oposición al panamericanismo. Ford ya se había referido a él en términos duros en diversos medios, incluso en su propio Main Currents of Spanish Literature, y Goldberg se había hecho eco de la mirada de su mentor en un artículo en el Boston Evening Standard. A pesar de todo eso, Goldberg y Blanco Fombona continuaron su correspondencia y sus tratos o intentos de negociar los derechos de las obras del segundo. Además, siguieron en conversaciones por Studies in Spanish-American Literature, que ya estaba a punto de publicarse. Sobre el libro, le dice Blanco Fombona a Goldberg:

Estoy deseosísimo de leer su trabajo sobre mí. No deje de enviármelo en cuanto salga el libro. Esto me resarcirá de las imbecilidades seniles del profesor Ford . . . quien piensa que yo puedo estar vendido a alguna potencia europea para atacar a los Estados Unidos. Por cierto, he leído . . . que acepta usted como probable, el extremo de que yo obedezca a propagandistas anti-yanquis. Advierto, por ese desliz de su pluma -así quiero calificarloque usted desconoce en absoluto mi sicología . . . Yo no soy hombre que se vende ${ }^{23}$ (Isaac Goldberg Papers).

Goldberg, en el fondo, sabe que Blanco Fombona no se vende. En su Studies in Spanish-American Literature el traductor lo llama "our most fanatic hater" (Gold-

22 J. M. D. Ford a Isaac Goldberg, 23 de julio de 1919. Isaac Goldberg Papers.

23 Rufino Blanco Fombona a Isaac Goldberg, octubre de 1919. Isaac Goldberg Papers. 
berg 1920: 336), pero al mismo tiempo declara respetarlo por su sinceridad a toda prueba. Además, se declara gran admirador de su versatilidad como escritor y le desea muchos años de abundante trabajo creativo, aunque también le desea que aprenda que no todo lo que viene de Estados Unidos es despreciable y poco digno de confianza. Goldberg se tensiona entre su admiración por el trabajo y la persona de Blanco Fombona y su lealtad por Estados Unidos y por el proyecto panamericanista. Finalmente, Goldberg solo traduce uno de los libros de Blanco Fombona, la novela El hombre de oro, pero la relación continúa y en 1922 América, la editorial del venezolano, publica la traducción al español de Studies in Spanish-American Literature ${ }^{24}$. No está claro si Goldberg no traduce más obras del venezolano debido a los vaivenes del mercado editorial y la popularidad de la literatura latinoamericana, que trataremos en el capítulo siguiente, o porque ya se acercaba el fin de su etapa traductora. Como sea, en el caso de El hombre de oro Goldberg traduce un texto que elige, pero lo hace dentro de un contexto claro, que pone a su traducción al servicio de las ambiciones del panamericanismo ${ }^{25}$. Esta traducción encarna una de las posibles relaciones entre la práctica de la traducción y el poder, como dice Edwin Gentzler, específicamente aquella en la cual la traducción viene a alimentar una idea nueva, el panamericanismo, que está en proceso de institucionalización. Pero, ¿cómo entender esta aparente tensión entre las obras que elige Goldberg y la causa que avanza su práctica de traductor? Goldberg desea un mundo cosmopolita, ese que presenta con añoranza cuando habla de su niñez en las calles de Boston. Quizá por lo mismo, se admira del deseo de unidad de la intelectualidad latinoamericana. Habla, en sus Studies, de que se está gestando una “All-America”, de que los países latinoamericanos están dando pasos para cumplir el sueño de Bolívar por lo menos en el área de las artes, de que está en ciernes una unidad de las mentes de la región ${ }^{26}$. Dice también que tal vez Latinoamérica es el porvenir del mundo (Goldberg 1920: 337). En esta idea de Goldberg convergen dos miradas sobre la región: la de las elites intelectuales y políticas de Estados Unidos que, desencantadas con la deshumanización de la modernidad, la ven como reserva de valores, y la mirada idealista que se da

24 La traducción al inglés del libro de Blanco Fombona se publica a fines de 1920, después de haber sido postergada para darle espacio en la apretada agenda traductora de Goldberg a una novela mexicana encargada por alguna editorial, según se desprende de la correspondencia de Goldberg y Blanco Fombona.

25 Tanto como con Studies in Spanish-American Literature (1920) como con Brazilian Literature (1922) Goldberg crea contexto para las traducciones de literatura contemporánea que planea hacer (ver capítulo 2).

26 Goldberg explica que all-America se refiere solo a la unidad de Latinoamérica, ya que, según dice, los latinoamericanos hablan de América para referirse solo a la región al sur del Río Grande. 
sobre la región, vista como un crisol de razas, desde ciertos sectores de la propia elite latinoamericana ${ }^{27}$.

Mi lectura es que el afán cosmopolita de Goldberg es lo que lo lleva a transitar los recorridos del panamericanismo, con los que tal vez, según propondrían un Ford o un mismo Goldberg, Estados Unidos podría incorporarse a esa unidad de mentes que se gesta en el sur. En esta idea hay, por cierto, una contradicción innata. Se propone, se desea, una unidad, un mundo universal de las mentes, de las letras, pero esa propuesta va de la mano con la idea de la superioridad de Estados Unidos, de su capacidad para liderar ese universalismo. Goldberg cree, al igual que Ford, que el intervencionismo estadounidense, las anexiones y las invasiones previas a la Política del Buen Vecino fueron obras de ciertos políticos corruptos y no del país. Cree, a pesar de todo, que Estados Unidos es el llamado a liderar el hemisferio. El de Goldberg es, entonces, un cosmopolitismo roto, una base fallida para sostener la unidad hemisférica -tal vez cree demasiado en las virtudes de la "American tradition”, de las que se declara enamorado en su texto sobre su infancia bostoniana, y por eso piensa que son las únicas que pueden liderar un proceso de unión del hemisferio. Por eso, es que desde allí sale en busca de un territorio común.

Goldberg dice en su estudio sobre los modernistas que es necesario saber lo que se piensa en Latinoamérica sobre Estados Unidos para así poder contestar y corregir ideas y acciones: "We must know the worst that Spanish America thinks of us and must strive to change that worst to best. If it takes two to make a quarrel, it takes two to make a friendship” (Goldberg 1920: 335-336). La necesidad de conocimiento como base de una relación con la que se abandera el panamericanismo se hace presente una vez más y, de nuevo, es la literatura la llamada a contestar preguntas. En este sentido, Goldberg cree en la traducción como una manera de anotar, de comentar. Como vimos en el capítulo anterior, la anotación y el comentario, son inherentes a la traducción. Si con sus traducciones Blackwell comentaba, corregía, el desequilibrio que veía en el intercambio cultural del hemisferio y con eso también al panamericanismo, Goldberg corrige paradigmas de representación para buscar la viabilidad de la unión regional. Él, como Henríquez Ureña más tarde, cree que el panamericanismo solo puede

27 Goldberg habla de la "nueva raza", la "nueva alma" que se gesta en Latinoamérica, resonando con la idea del mestizaje como fortaleza de la región que sintetizará José Vasconcelos solo cinco años después en La raza cósmica. A mediados del siglo XIX, las elites intelectuales latinoamericanas comienzan a preguntarse por el significado de "Latinoamérica" y le encuentran sentido a la categoría en la idea del mestizaje asociado a una sociedad fundada en las ideas antes que en el consumo, en los valores antes que en la competitividad económica y con más énfasis en el cuerpo social que en el individuo. Para más sobre esto ver Friedman (2012). 
ser viable si primero hay un cambio en las miradas de Estados Unidos sobre Latinoamérica y vice versa. Por eso despliega a los modernistas en su libro, explica las influencias y las conexiones con Francia, los contextualiza en sus ideas políticas, económicas, en su mirada a Estados Unidos. Y aunque a veces él mismo cae en los estereotipos que intenta reescribir -como cuando dice que los latinoamericanos nacen recitando poesía y, por lo tanto, crecen propensos a la exageración- los incluye junto a europeos y a poetas neoyorquinos, así en general, sin nombrar ninguno, como parte de un mismo fenómeno que trasciende bordes y distancias geopolíticas: la modernidad. Así, también, inscribe a estos escritores latinoamericanos en ese mundo cosmopolita en el que dice habitar -o que desea habitar- desde niño.

\section{As Latin America Sees Us}

La propuesta de Goldberg, como la de sus colegas panamericanistas, tiene que ver con leer al vecino para conocerlo, incorporarlo, y lograr establecer una alianza regional. Su herramienta es la literatura -la traducción como parte de ella, por un tiempo-, porque cree en la palabra, en el milagro de las letras, como también cree en la superioridad de Estados Unidos y en la necesidad de cambiar los paradigmas de representación, como dijimos anteriormente. Hay que limpiar la mente de preconcepciones, dice ahí Goldberg, quien revisita el tema de las miradas en un artículo llamado “As Latin America Sees Us” (Cómo nos ve Latinoamérica). "Physical and material inferiority, as everyone knows, has a habit of translating itself into a sense of spiritual superiority" (Goldberg 1924: 465), dice Goldberg, quien luego explica que de esa supuesta superioridad Latinoamérica ha sacado la idea de que tiene una noble misión que cumplir en el hemisferio occidental, una que cobra dimensión cuando es contrapuesta a cierta imagen de Estados Unidos:

\footnotetext{
We of the North are visioned as the incarnation of a gross materialism while they of the South become paladins of idealism. We are vultures, birds of prey; they are swans, symbols of grace and culture. We are heavy-footed prose; they are light-wing poesy. These are all lies of self-defense, but in them dwells a core of truth. Translate these metaphors into the language of contemporary economics, and they mean simply that, with the sure instinct of the weaker, the South Americans sense in us a predestined enemy (el énfasis es mío, Goldberg 1924: 465).
}

Goldberg presenta la visión de Latinoamérica sobre Estados Unidos usando sus lecturas de Darío, de Rodó, y luego explica que deplora la idea del coloso del norte a punto de caer sobre los países del sur y que solo puede esperar que aquel temor no sea más que un espejismo, uno levantado sobre el desconocimiento y la 
expansión de los estereotipos sobre el otro. Lo que él ve, es un territorio común. Es haciendo una lectura del Ariel -“A remarkable essay, for all the gaudy attire in which it moves” (Goldberg 1924: 466)-, donde Goldberg entrega pistas de esa unidad que presiente: "Ariel and Caliban are nor neat separable entities; they are twin aspects of a single continental personality; whether above or below the Rio Grande" (el énfasis es mío, Goldberg 1924: 467). Si fuera una sola la personalidad que existe a lo largo del continente tal vez no sería tan difícil hacer que el deseo de esa "all-America” que Goldberg lee en los intelectuales latinoamericanos pudiera incluir a Estados Unidos, a sus intelectuales. La Pan-América deseada desde el norte. Por eso el empeño de Goldberg por derrotar al desconocimiento mutuo:

On our own side, we are, even when not frankly belligerent or diplomatically oblique, just as one-sided in our views as the South Americans. Our moving-picture Mexican is hardly an exaggeration of what the popular mind, by the newspapers and other agencies, has been taught to regard as the Latin-American reality . . . a nightmare of bull-fights, bandits, revolutions, guitars and ogling señoritas . . . By way of return compliment we . . . become a complex of money grubbing swindlers, materialists with the hides of rhinoceroses, divorcing our wives every Monday and Thursday, lynching Negroes between dinner courses, and lighting our cigars with thousand-dollar bills (Goldberg 1924: 465).

Goldberg es consciente de la manera en que las representaciones sobre Latinoamérica se han construido en Estados Unidos. Uno de los culpables que señala es la prensa, las letras rápidas, efímeras, que sin embargo van construyendo imaginarios duraderos. Su propuesta entonces es la de las letras lentas, usar la literatura que se queda en el tiempo y va construyendo archivo de conocimiento para vencer imaginarios tan manoseados. Por esto, en este artículo, Goldberg intenta un mapa de las lecturas latinoamericanas de la escritura estadounidense. ¿Qué saben sobre nosotros?, se pregunta. ¿Qué han leído? Así recorre los “ABC countries” y después da un vistazo más general a varios otros países de la región. Se conoce algo de oídas, se ha leído poco, más que nada a Poe, muchas veces en francés o en algunas de sus traducciones al español, y Whitman, algo traducido. Después, según los países, suenan, más o menos, algunos nombres como los de Emerson, Thoreau, Mark Twain y Amy Lowell ${ }^{28}$. Lo que Goldberg dice explícitamente sobre Argentina vale para toda la región: lo poco que se lee de literatura estadounidense se lee, sobre todo, en español o en francés, "readers

28 En Argentina parece haber un mayor conocimiento de las letras estadounidenses -que incluye nombres como el de Jack London $\mathrm{u}$ O. Henry- gracias a las traducciones que publica la prensa, especialmente La Nación. Aquí, Goldberg le da crédito al periodista y escritor chileno Ernesto Montenegro por escribir para La Nación numerosos artículos y algunas traducciones sobre escritores y escritura estadounidense (Goldberg 1924: 469). 
who know English are rarities" (Goldberg 1924: 468). En el sentido inverso y a pesar de toda la popularidad de la enseñanza del español, pasa algo parecido. La enseñanza de las lenguas extranjeras está muy lejos de poder conformar masas lectoras en el idioma enseñado. Cuenta Fernando Degiovanni que años después, ya en los 40, Américo Castro, desde su puesto en Princeton, hablará sobre la inconveniencia de enseñar español a estudiantes estadounidenses con textos del modernismo y del regionalismo. Castro dirá que si bien estos textos son útiles para aprender sobre "la vida y el carácter hispanoamericanos" (Degiovanni 2016: 2014) -es decir lo mismo que han dicho Coester, Goldberg y otros- la dificultad lingüística que presentan es irremontable para los estudiantes. Aunque el filólogo español tenía su propia agenda en mente al hacer estos comentarios -trabajar el tema del "entendimiento" panamericano o en su caso "el desentendimiento", para darle centralidad al estudio de la cultura y literatura española- apuntaba a un tema clave, como dice Degiovanni: "La construcción de una comunidad lectora hemisférica a partir de la adquisición de una segunda lengua tropieza así con sus propias promesas de igualdad y accesibilidad” (Degiovanni 2016: 214). Goldberg, en su “As Latin America Sees Us” había apuntado en la misma dirección al notar la incapacidad de leer inglés que se extendía por Latinoamérica. El traductor presenta a una de las fuentes de su artículo, el escritor argentino Manuel Gálvez, como alguien "who reads English” y que por lo tanto está mejor informado sobre la literatura estadounidense que la mayoría de los latinoamericanos ${ }^{29}$. Gálvez, desde el lugar de confianza que le otorga su manejo del inglés, le confirma a Goldberg que en Latinoamérica se lee muy poco de la literatura de Estados Unidos y que se cree, en general, que los norteamericanos solo piensan en amasar fortunas. También le dice:

You Americans are to blame for our unfavorable attitude. You take no interest in us and I believe this is to be deplored. Our literatures and our writers would gain mutual understanding. . . One thing alone is needed. . . that your books be translated and published in Spain (El énfasis es mío, Goldberg 1924: 468).

Traducción. Ese es el camino para la literatura extranjera ante la imposibilidad de crear masas lectoras. Y Goldberg se aboca a eso porque:

Minds, like water, seek a common level. The natural channel is print . . .What is needed for the immediate present . . . [is] a purely aesthetic consideration of each other's accom-

29 Dice Goldberg que Gálvez es el Upton Sinclair, también bastante leído en Latinoamérica, de Argentina. Goldberg intentó conseguir los derechos de traducción de Nacha Regules, pero Gálvez se disculpó por dárselos a una editorial -Dutton-, que ya tenía traductor. Gálvez le explicó a Goldberg que había decidido hacer eso porque Dutton le ofrecía paga, poca, pero suficiente. 
plishment . . . The Americas have nothing to lose by closer communion, and commercial advantage is the least of the things to be gained (Goldberg 1924: 471).

La comunión a través de los textos, eso dice el apóstol del panamericanismo. Así, se aboca a la traducción y a la difusión de las letras latinoamericanas hasta que la traducción cumple su ciclo y él pasa a otra cosa, a escribir una biografía de George Gershwin, el volumen de cuentos Sexarian, una biografía sobre Lola Montes, un libro sobre los protocolos de los sabios de Sion, otro sobre intelectuales que vuelven a caer en las garras de la religión en tiempos de crisis internacionales. Goldberg escribe hasta que una enfermedad repentina ya no lo deja. De todas maneras, antes de eso, ya para comienzos de los 30, cuando acepta el puesto en Harvard, parecía haber dejado de esperar que alguna forma de su escritura o sus pasiones le llenara los bolsillos. Luego de comenzar a enseñar la clase que le había ofrecido Ford en Harvard en 1932, Goldberg se ríe de verse de vuelta en la academia, de estar disfrutándola ${ }^{30}$. Además, escribe que en el tiempo libre que le deja la enseñanza -y al decir "spare time" se asume amateur en su escritura, como no lo fue en la traducción-, hace lo que más le gusta, ordena sus notas sobre literatura latinoamericana pensando en publicarlas como un par de libros más, piensa algún día en escribir para el cine y prepara el lanzamiento de su revista Panorama; A Monthly Survey of People and Ideas. Dice sobre ella:

It will not make money, perhaps; it will disseminate ideas, and that too, is valuable coinage. It will be devoted, need I add, to the expression of an international outlook, -to the more or less universal language of thought and emotion that exists behind the various dictionaries of mankind (Goldberg 1933: 273).

Goldberg es entonces, al final de todo, un profesional de las letras y un cosmopolita, aunque su idea de cosmopolitismo esté quebrada. Sus intentos por hacer de la traducción una profesión que le diera para vivir lo llevaron a perder por momentos su libertad de elección y el compromiso con el material a traducir, el uso de su tiempo, incluso la misma calidad del trabajo que amaba, de esa escritura de los bordes que es también la traducción. Como dijimos en el capítulo anterior uno de los factores que hace del amateurismo una condición permanentemente marginal es la valoración social que se hace del amateur, la mirada peyorativa sobre su formación, sobre sus habilidades. Esta mirada que anula al amateur contraponiéndolo con el profesional anula la posibilidad de que el primero sea remunerado. Goldberg anuló a Goldberg amateur. Tal vez su esfuerzo

30 "As the course is not a linguistic one, but rather an aspect of comparative literature and the aesthetics of literary forms, I have enjoyed it immensely", contaría después Goldberg (Goldberg 1933: 273). 
debería haber sido otro, no el de buscar la profesionalización a través de horas, plazos de entrega, cantidades imposibles de trabajo hecho a toda velocidad. Tal vez hubiera debido mantener la relación de su práctica traductora dentro del territorio del amateur, es decir, en relación con el placer, con el gusto de elegir los trabajos de traducción que quería y haber negociado la mejor paga posible por eso, y haber vivido de alguna otra actividad relacionada con su escritura o formación. Aunque tal vez esto no le hubiera interesado.

Hace un tiempo le preguntaron a Michael Hofmann, poeta y traductor, si en esta etapa de su carrera, con más de 80 libros traducidos desde el alemán al inglés, era particularmente selectivo con los textos que elegía trabajar. Hofmann contestó que siempre había que comenzar por alguna parte y que el siempre comenzaba por ser susceptible de ser contratado, pero que era importante "to have that little word "No" somewhere around. Your parachute. Not everything deserves to be translated. Not everything should be translated" (Hofmann 2017). Tal vez ese no/paracaídas le hubiera permitido a Goldberg negociar de otra manera su faceta de traductor, aunque también cabe la posibilidad de que esa etapa de su escritura se hubiera cerrado sin importar los arreglos llevados a cabo. Como sea, durante su trabajo como traductor Goldberg se movió fuera del territorio amateur. A veces, hizo lo que deseaba y eligió textos a los que dedicó, tal vez, más recursos y tiempo. También se hizo parte de ese engranaje que puso en movimiento, de otra manera, haciendo sus propias correcciones, la circulación de productos culturales que no tenían antes un espacio en la cultura receptora, en el mercado literario de Estados Unidos. Tal vez, de alguna manera, el Goldberg traductor encarnó de manera torcida esa conversación constante entre amateurismo y profesionalismo. Como sea, como traductor Goldberg también corrigió, anotó, buscó demoler paradigmas representacionales en pos de una unidad intelectual que aunque movida directamente por el panamericanismo, en su caso se inspiraba en el cosmopolitismo, uno roto, pero que de todas maneras creía en una posible unidad regional, aunque solo fuera, como decía él, en el territorio de las artes. 
